\title{
AN INTEGRAL EQUATION INVOLVING LEGENDRE FUNCTIONS*
}

\section{A. ERDÉLYI $\dagger$}

1. Introduction. In recent years there appeared solutions of several integral equations whose kernels contain one of the classical orthogonal polynomials. $\mathrm{Ta} \mathrm{Li}[1]$ solved the integral equation

$$
\int_{\sigma}^{1}\left(u^{2}-\sigma^{2}\right)^{-\frac{1}{2}} T_{n}\left(\frac{u}{\sigma}\right) y(u) d u=f(\sigma), \quad 0<c \leqq \sigma \leqq 1,
$$

which arose in the solution of a certain aerodynamical problem and in which $T_{n}$ is the Chebyshev polynomial of the first kind; R. G. Buschman [2] found the solution of the analogous integral equation

$$
\int_{\sigma}^{1} P_{n}\left(\frac{u}{\sigma}\right) y(u) d u=f(\sigma), \quad 0<c \leqq \sigma \leqq 1,
$$

involving the Legendre polynomial $P_{n}$; and T. P. Higgins [3] discussed an integral equation which includes both (1.1) and (1.2) as special cases, namely the equation

$$
\int_{\sigma}^{1}\left(u^{2}-\sigma^{2}\right)^{\lambda-\frac{1}{2}} C_{n}^{\lambda}\left(\frac{u}{\sigma}\right) y(u) d u=f(\sigma), \quad 0<c \leqq \sigma \leqq 1,
$$

in which $C_{n}{ }^{\lambda}$ is an ultraspherical (Gegenbauer) polynomial. ${ }^{1}$

The solution of these integral equations by the aforementioned authors is based on certain integrals involving products of the respective orthogonal polynomials. Another method of solution can be based on Rodrigues's formula and its extension to the classical orthogonal polynomials. By means of this formula it is possible to represent the left hand side of the integral equation as a derivative of order $n$ of an integral whose inversion is a problem in integral equations of Abel's type - and therefore a known problem.

This second method of solution can be presented in a slightly different form which is especially simple in the case of Buschman's integral equation. The left hand side of (1.2) is a constant multiple of the derivative of order

* Received by the editors March 15, 1963.

$\dagger$ Department of Mathematics, California Institute of Technology, Pasadena, California. The preparation of this paper was partly supported by the National Science Foundation under Grant No. GP-213 to the California Institute of Technology.

1 The author is indebted to Dr. Higgins for permission to utilize the typescript of the latter's paper before publication, and also for obtaining access to further unpublished work. 
$n$ with respect to $\sigma^{-1}$ of

$$
\sigma^{-2 n} \int_{\sigma}^{1}\left(u^{2}-\sigma^{2}\right)^{n} u^{-n} y(u) d u
$$

and the integral appearing here is a multiple of the $n+1$ times repeated integral with respect to $\sigma^{2}$ of $\sigma^{-n-1} y(\sigma)$. This observation leads to an elementary solution [4] of (1.2) under conditions which are both necessary and sufficient for the existence of an integrable solution $y(u)$ of (1.2).

Rodrigues's formula can be applied also to (1.1) and (1.3) but here the situation is slightly more involved in that the integrals with respect to $\sigma^{2}$ are of fractional order and their inversion requires the knowledge of differentiation and integration of fractional order. ${ }^{2}$ In spite of this complication the method has its merits and seems more direct than that employed in [1] and [3]. Moreover, once differentiation and integration of fractional order are used, it seems appropriate to allow a derivative of fractional order with respect to $\sigma^{-1}$ to appear so that the ultraspherical polynomial in (1.3) may be replaced by an (associated) Legendre function. This will be done in the present paper.

Although this slight generalization and the resulting form of the solution which differs from that obtained in [1], [2], [3] and in some cases entails more liberal conditions of validity, are of some interest in themselves, the motivation for this paper lies not so much in these (slight) improvements on known results as in the desire to illustrate the usefulness of fractional integrals as a tool in applied mathematics. With this in mind, the descriptive account (in \$2) of fractional integrals and derivatives and their application (in \$3) to Legendre functions are given in a little more detail than is required for the solution of the integral equation.

In $\$ 4$, the integral equation

$$
\begin{aligned}
& \int_{a}^{x}\left(x^{2}-t^{2}\right)^{\lambda / 2} P_{\nu}^{-\lambda}\left(\frac{x}{t}\right) g(t) d t=f(x), \\
& \lambda>-1, \quad \nu \geqq-\frac{1}{2}, \quad 0<a \leqq x \leqq b,
\end{aligned}
$$

is solved in the form

$$
g(x)=(2 x)^{\nu+1} I_{x^{2}}^{-\nu-1} I_{x}^{\nu-\lambda} f(x)
$$

(for the definition of the operator $I^{\alpha}$ see $\$ 2$, for the conditions of validity

2 Derivatives of fractional order appear in certain cases also in Higgins's work in an intermediate step leading towards the solution, and the same author applied fractional differentiation to an integral equation whose kernel contains a hypergeometric function. [Note added September, 1963: This work has been prepared for publication under the title, A hypergeometric function transform.] 
see $\$ 4)$; and in $\$ 5$ explicit analytic forms of this solution are obtained in various cases depending on whether $\nu$ and $\lambda-\nu$ are or are not nonnegative integers. Better, if less explicit, conditions of validity and an alternative form of the solution are obtained in $\S 6$.

2. Fractional integration and differentiation. Let $[a, b]$ be a compact (bounded and closed) real interval; let $A_{0}$ be the class of complex-valued functions integrable on $[a, b]$; and for positive integer $k$ let $A_{k}$ be the class of functions for which $(i) f^{(k-1)}(x)$ exists and is absolutely continuous on $[a, b]$ and (ii)

$$
f(a)=f^{\prime}(a)=\cdots=f^{(k-1)}(a)=0 .
$$

Two functions equal almost everywhere (that is, equal except on a set of measure zero) are not regarded as distinct, and for functions belonging to $A_{0}$ it is sufficient to be defined almost everywhere (a.e.). $f^{(j)}$ indicates the $j$ th derivative of $f$. Since $f^{(k-1)}(x)$ is absolutely continuous, $f^{(k)}(x)$ exists a.e. and defines an integrable function.

Let $I_{x}$ be the operator of integration defined by

$$
I_{x} f(x)=\int_{a}^{x} f(t) d t
$$

so that the operation of $k$ times repeated integration is expressed as

$$
I_{x}^{k} f(x)=\frac{1}{(k-1) !} \int_{a}^{x}(x-t)^{k-1} f(t) d t, \quad k=1,2,3, \cdots ;
$$

and set

$$
I_{x}^{0} f(x)=f(x), \quad I_{x}^{-k} f(x)=f^{(k)}(x), \quad k=1,2, \cdots .
$$

The most important properties of $I_{x}{ }^{k}$ may be summarized as follows. If $f \in A_{j}, j=0,1,2, \cdots$, and $k$ is an integer (positive, negative, or zero) for which $j+k \geqq 0$, then $I_{x}^{k} f$ exists and belongs to $A_{j+k}$; if $l$ is a further nonnegative integer which does not exceed $j+k$, then

$$
\left(\frac{d}{d x}\right)^{l} I_{x}^{k} f(x)=I_{x}^{k-l} f(x)
$$

exists and belongs to $A_{j+k-l}$. Also, for $f \in A_{k}, k=0,1,2, \cdots$, we have

$$
f(x)=I_{x}^{k} f^{(k)}(x) .
$$

We shall now extend these and other results to nonintegral values of the index. For the sake of simplicity we restrict ourselves to real indices although complex indices with nonzero real part are easily included, and 
imaginary indices have also been considered [5]. Accordingly, $\alpha, \beta, \cdots$, will be real numbers, and, as usual, $[\alpha]$ will stand for the largest integer not exceeding $\alpha$.

For $\alpha>0$, we follow Riemann and Liouville in defining the integral of order $\alpha$ of $f$ as

$$
I_{x}^{\alpha} f(x)=\frac{1}{\Gamma(\alpha)} \int_{a}^{x}(x-t)^{\alpha-1} f(t) d t .
$$

By a simple application of Fubini's theorem it can be shown that $I_{x}{ }^{\alpha} f(x)$ exists a.e. and defines an integrable function if $f$ is integrable and $\alpha>0$. The further formula

$$
I_{x}^{\alpha}\left(I_{x}^{\beta} f\right)(x)=I_{x}^{\alpha+\beta} f(x)
$$

for $f \in A_{0}, \alpha>0, \beta>0$ can be proved by interchanging the order of integrations (again by Fubini's theorem) in the repeated integral indicated on the left hand side of (2.8).

For $\alpha \geqq 1$ (and $f \in A_{0}$ ), $I_{x}{ }^{\alpha}$ is not merely integrable but absolutely continuous, and

$$
\frac{d}{d x} I_{x}^{\alpha} f(x)=I_{x}^{\alpha-1} f(x) .
$$

This is immediate for $\alpha=1$; for $\alpha>1$ it can be proved by differentiation "under the integral sign", a process justifiable by Lebesgue's theorem on dominated convergence.

If $\alpha<0$, we define $I_{x}^{\alpha} f$ indirectly, saying that $I_{x}^{\alpha} f=g$ if $f=I_{x}^{-\alpha} g$. $I_{x}{ }^{\alpha} f$ for $\alpha<0$, if it exists, is thus defined up to a function vanishing a.e.. It is easy to verify that for the so extended operator $I_{x}{ }^{\alpha}$, for all real $\alpha$, (2.8) and (2.9) hold provided that the functions involved in these relations make sense. Moreover, this definition of $I_{x}{ }^{\alpha}$ agrees, in the case of integer $\alpha$, with the definition given at the beginning of this section. For negative $\alpha, I_{x}{ }^{\alpha}$ is an operator of differentiation of fractional order but it will be convenient not to make this distinction: we regard differentiation as integration of negative order and conversely, we consider integration as differentiation of negative order.

If $f \in A_{k}, k=1,2, \cdots$, and $-k \leqq \alpha<0$, then

$$
f(x)=I_{x}^{k} f^{(k)}(x)=I_{x}^{-\alpha}\left(I_{x}^{k+\alpha} f^{(k)}\right)(x)
$$

by (2.6) and (2.8). It follows that for $f \in A_{k}$ and $\alpha+k \geqq 0, I_{x}{ }^{\alpha} f(x)$ exists, and

$$
I_{x}^{\alpha} f(x)=I_{x}^{\alpha+k} f^{(k)}(x) .
$$

Combining these various pieces of information, we can now show that 
for $f \in A_{k}$ and $\alpha+k \geqq 0, I_{x}^{\alpha} f$ exists and belongs to $A_{k+[\alpha]}$; and if $j$ is a nonnegative integer not exceeding $k+[\alpha]$, then

$$
\left(\frac{d}{d x}\right)^{j} I_{x}^{\alpha} f(x)=I_{x}^{\alpha-j} f(x)=I_{x}^{\alpha-j+k} f^{(k)}(x) .
$$

Moreover, (2.8) holds for $f \in A_{k}$ provided $[\beta]+k \geqq 0$ and $[\alpha]+[\beta]+k \geqq 0$.

For the sake of simplicity we considered the operators $I_{x}{ }^{\alpha}$ on $A_{k}$. It should be understood that the conditions given above for the existence of $I_{x}^{\alpha} f$, while sufficient, are by no means necessary, and that the statements made above with regard to continuity properties are certainly not the best possible statements. For instance, it is known [6, Theorem 4] that for $-1<\alpha<0, I_{x}^{\alpha} f$ exists as an integrable function if and only if $f \in A_{0}$ and $I_{x}{ }^{\alpha+1} f(x)$ is an absolutely continuous function vanishing at $a$. This is a much less restrictive condition than $f \in A_{1}$, but also one that is more difficult to verify. For more effective results and conditions it seems appropriate to consider functions subject to Lipschitz conditions [7] but we shall not discuss this refinement here.

If $a>0$ so that $x=0$ is not a point of $[a, b]$, we can define also fractional integration with respect to $x^{n}$ for any nonzero real $n$. For $\alpha>0$, the appropriate definition is

$$
I_{x^{n}}^{\alpha} f(x)=\frac{1}{\Gamma(\alpha)} \int_{a}^{x}\left(x^{n}-t^{n}\right)^{\alpha-1} f(t) n t^{n-1} d t,
$$

and the extension to $\alpha \leqq 0$ is made as before. The analogues of the basic identities remain valid. In particular,

$$
I_{x^{n}}^{\alpha} I_{x^{n}}^{\beta}=I_{x^{n}}^{\alpha+\beta}
$$

remains valid so that $I_{x^{n}}^{\alpha}$ and $I_{x^{n}}^{\beta}$ commute. Note, however, that for $m \neq n$, $I_{x^{m}}^{\alpha}$ and $I_{x^{n}}^{\beta}$ do not commute. Since $x$ and $x^{n}$ are indefinitely differentiable functions of one another on $[a, b]$ if $a>0$, all continuity and integrability properties are preserved when the variable is changed, and the operator $I_{x^{n}}^{\alpha}$ can be defined on the classes $A_{k}$ that are independent of $n$.

3. Legendre functions. It is appropriate to use a different normalization of Legendre functions according as the variable is restricted to the interval $(-1,1)$ (the "cut") or varies in the complex plane (in the first instance slit along the real axis from $-\infty$ to 1 ). In this paper, the variable appearing in the Legendre functions will always be positive real and the two normalizations will be used respectively, on $(0,1)$ and $(1, \infty)$. The functions themselves will be distinguished as $\mathrm{P}_{\nu}{ }^{\lambda}(z)$, for $-1<z<1$, and $P_{\nu}{ }^{\lambda}(z)$, for $z>1$ or complex $z$. 
In the first instance $P_{\nu}^{-\lambda}(z)$ is defined in terms of a hypergeometric series $[8, \S 3.2(16)]$ but we shall replace this series by the Eulerian integral representing it $[8, \S 2.1(10)]$, defining

$$
\begin{aligned}
& P_{\nu}^{-\lambda}(z)=\frac{\{(z-1) /(z+1)\}^{\lambda / 2}}{2^{\nu} \Gamma(\nu+1) \Gamma(\lambda-\nu)} \\
& \cdot \int_{0}^{1} v^{\lambda-\nu-1}(1-v)^{\nu}\{z+1-(z-1) v\}^{\nu} d v, \quad \lambda>\nu>-1, z>1
\end{aligned}
$$

and

$$
\begin{aligned}
& \mathrm{P}_{\nu}^{-\lambda}(z)= \frac{\{(1-z) /(1+z)\}^{\lambda / 2}}{2^{\nu} \Gamma(\nu+1) \Gamma(\lambda-\nu)} \\
& \cdot \int_{0}^{1} v^{\lambda-\nu-1}(1-v)^{\nu}\{z+1-(z-1) v\}^{\nu} d v, \\
& \lambda>\nu>-1,-1<z<1 .
\end{aligned}
$$

Here all fractional powers are given their principal (in this case positive) values. The restriction to real values of $\lambda, \nu, z$ is inessential.

The following recurrence and reduction formulas will be needed in the sequel.

$$
\begin{array}{cr}
P_{\nu}^{-\lambda}(z)=P_{-\nu-1}^{-\lambda}(z) & z>1, \\
P_{\nu}^{-\nu}(z)=P_{-\nu-1}^{-\nu}(z)=\frac{\left(z^{2}-1\right)^{\nu / 2}}{2^{\nu} \Gamma(\nu+1)}, & -1<z<1, \\
P_{0}^{-\lambda}(z)=\frac{1}{\Gamma(\lambda+1)}\left(\frac{1-z}{1+z}\right)^{\lambda / 2}, & -1<z<1 .
\end{array}
$$

These are known $[8, \S 3.3,3.6,3.8]$.

We shall now establish two results on fractional integrals of Legendre functions. In these, $a>0, t$ and $x$ vary over the interval $[a, b]$, and $H$ is Heaviside's unit function defined by

$$
H(u)=1 \text { if } u>0, \quad H(u)=0 \text { if } u<0 .
$$

The first result $[9, \S 13.1(52)]$ is

$$
\begin{aligned}
I_{x}^{\alpha}\left\{\left(x^{2}-t^{2}\right)^{\lambda / 2} P_{\nu}^{-\lambda}\left(\frac{x}{t}\right) H(x-t)\right\} & \\
& =\left(x^{2}-t^{2}\right)^{(\lambda+\alpha) / 2} P_{\nu}^{-\lambda-\alpha}\left(\frac{x}{t}\right) H(x-t)
\end{aligned}
$$

and holds when either $\alpha \geqq 0$ and $\lambda>-1$ or $\alpha \leqq 0$ and $\lambda>-\alpha-1$. 
In order to prove (3.7), we consider for $\alpha>0, \beta>0$, and $a \leqq t<x \leqq b$, $I_{x}^{\alpha}\left\{\frac{H(x-t)}{\Gamma(\beta)}\left(x^{2}-t^{2}\right)^{\beta-1}\right\}=\frac{1}{\Gamma(\alpha) \Gamma(\beta)} \int_{t}^{x}(x-u)^{\alpha-1}\left(u^{2}-t^{2}\right)^{\beta-1} d u$.

Here we introduce a new variable of integration $v$ by the substitution $u=x-(x-t) v$ and obtain

$$
\frac{1}{\Gamma(\alpha) \Gamma(\beta)}(x-t)^{\alpha+\beta-1} \int_{0}^{1} v^{\alpha-1}(1-v)^{\beta-1}\{x+t-(x-t) v\}^{\beta-1} d v .
$$

On account of (3.1) we thus have

$$
\begin{aligned}
I_{x}^{\alpha}\left\{\frac { H ( x - t ) } { \Gamma ( \beta ) } \left(x^{2}\right.\right. & \left.\left.-t^{2}\right)^{\beta-1}\right\} \\
& =(2 t)^{\beta-1}\left(x^{2}-t^{2}\right)^{(\alpha+\beta-1) / 2} P_{\beta-1}^{1-\alpha-\beta}\left(\frac{x}{t}\right) H(x-t)
\end{aligned}
$$

for $\alpha \geqq 0, \beta>0$. In view of the definition of $I_{x}{ }^{\alpha}$ for $\alpha<0$ we also have

$$
\begin{aligned}
I_{x}^{\nu-\lambda}\left\{\left(x^{2}-t^{2}\right)^{\lambda / 2} P_{\nu}^{-\lambda}\left(\frac{x}{t}\right) H(x\right. & -t)\} \\
& =\frac{H(x-t)}{\Gamma(\nu+1)}(2 t)^{-\nu}\left(x^{2}-t^{2}\right)^{\nu}
\end{aligned}
$$

for $\lambda \geqq \nu>-1$.

Returning now to (3.7), we note that on account of (3.3) we may take $\nu \geqq-\frac{1}{2}$. If $\lambda>\nu \geqq-\frac{1}{2}$ and $\alpha>0$, then the left hand side of (3.7) is

$$
I_{x}^{\alpha} I_{x}^{\lambda-\nu}\left\{\frac{H(x-t)}{\Gamma(\nu+1)}(2 t)^{-\nu}\left(x^{2}-t^{2}\right)^{\nu}\right\}
$$

by (3.8). Here $I_{x}{ }^{\alpha} I_{x}{ }^{\lambda-\nu}=I_{x}{ }^{\alpha+\lambda-\nu}$ by (2.8), whereupon a second application of (3.8) leads to the right hand side of (3.7). Thus, this relation is now established for $\alpha>0, \lambda>\nu \geqq-\frac{1}{2}$. Holding $\alpha$ and $\nu$ fixed, we see that both sides are analytic functions of the complex parameter $\lambda$, regular when $\operatorname{Re} \lambda>-1$. Thus, (3.7) holds when $\alpha>0, \lambda>-1, \nu \geqq-\frac{1}{2}$. The extension to $\alpha=0$ is trivial, and the extension to $\nu<-\frac{1}{2}$ follows from (3.3), since $-\nu-1>-\frac{1}{2}$ in this case; so (3.7) holds when $\alpha>0$ and $\lambda>-1$. From this it follows that

$$
\begin{aligned}
I_{x}^{-\alpha}\left\{\left(x^{2}-t^{2}\right)^{(\lambda+\alpha) / 2} P_{\nu}^{-\lambda-\alpha}\left(\frac{x}{t}\right)\right. & H(x-t)\} \\
= & \left(x^{2}-t^{2}\right)^{\lambda / 2} P_{\nu}^{-\lambda}\left(\frac{x}{t}\right) H(x-t)
\end{aligned}
$$


if $\alpha \leqq 0$ and $\lambda+\alpha>-1$, and in view of the definition of $I_{x}{ }^{\alpha}$ for $\alpha<0$ this proves the validity of (3.7) under the conditions $\alpha \leqq 0, \lambda>-\alpha-1$.

Lastly, putting $\nu=\lambda=\beta-1$ in (3.7) and using (3.4) we find that (3.8) holds not only when $\alpha \geqq 0$ and $\beta>0$ but also when $\alpha \leqq 0$ and $\alpha+\beta>0$; and putting $\alpha=\nu-\lambda$ in (3.7) and using (3.4) we find that (3.9) holds not only when $\lambda \geqq \nu>-1$ but also when $\nu \geqq \lambda>-1$.

(3.8) may be considered as an extension to "Gegenbauer functions" $[8, \$ 3.15 .2]$ of Rodrigues' formula for Gegenbauer polynomials $[8, \S 3.15(10)]$; we obtain the latter formula if $\alpha=-n, \beta=n+\lambda+\frac{1}{2}$, $n=0,1,2, \cdots$, in (3.8).

The second result on fractional integrals of Legendre functions is

$$
\begin{aligned}
I_{x^{2}}^{\alpha}\left\{(2 x)^{\nu}\left(x^{2}-t^{2}\right)^{\lambda / 2}\right. & \left.\mathrm{P}_{\nu}^{-\lambda}\left(\frac{t}{x}\right) H(x-t)\right\} \\
& =(2 x)^{\nu+\alpha}\left(x^{2}-t^{2}\right)^{(\lambda+\alpha) / 2} \mathrm{P}_{\nu+\alpha}^{-\lambda-\alpha}\left(\frac{t}{x}\right) H(x-t),
\end{aligned}
$$

and it too holds if either $\alpha \geqq 0$ and $\lambda>-1$ or $\alpha \leqq 0$ and $\lambda>-\alpha-1$.

To prove this, we assume $\alpha>0, \beta>0, a \leqq t<x \leqq b$ and consider

$$
I_{x^{2}}^{\alpha}\left\{\frac{H(x-t)}{\Gamma(\beta)}(x-t)^{\beta-1}\right\}=\frac{2}{\Gamma(\alpha) \Gamma(\beta)} \int_{t}^{x}\left(x^{2}-u^{2}\right)^{\alpha-1}(u-t)^{\beta-1} u d u .
$$

With $u=t+(x-t) v$ this becomes

$$
\begin{aligned}
& \frac{2 t}{\Gamma(\alpha) \Gamma(\beta)}(x-t)^{\alpha+\beta-1} \int_{0}^{1} v^{\beta-1}(1-v)^{\alpha-1}\{x+t-(t-x) v\}^{\alpha-1} d v \\
& +\frac{2}{\Gamma(\alpha) \Gamma(\beta)}(x-t)^{\alpha+\beta} \int_{0}^{1} v^{\beta}(1-v)^{\alpha-1}\{x+t-(t-x) v\}^{\alpha-1} d v .
\end{aligned}
$$

This is

$$
2^{\alpha} x^{\alpha-1}\left(x^{2}-t^{2}\right)^{(\alpha+\beta-1) / 2}\left\{t \mathrm{P}_{\alpha-1}^{1-\alpha-\beta}\left(\frac{t}{x}\right)+\beta\left(x^{2}-t^{2}\right)^{1 / 2} \mathrm{P}_{\alpha-1}^{-\alpha-\beta}\left(\frac{t}{x}\right)\right\}
$$

in view of (3.2). Here we can use (3.6) and obtain

$$
\begin{aligned}
I_{x^{2}}^{\alpha}\left\{\frac{H(x-t)}{\Gamma(\beta)}(x-\right. & \left.t)^{\beta-1}\right\} \\
& =(2 x)^{\alpha}\left(x^{2}-t^{2}\right)^{(\alpha+\beta-1) / 2} \mathrm{P}_{\alpha}^{1-\alpha-\beta}\left(\frac{t}{x}\right) H(x-t) .
\end{aligned}
$$

From this result (3.10) can be deduced by steps analogous to those leading 
from (3.8) to (3.7). The relation analogous to (3.9) in this case is

$$
\begin{aligned}
I_{x^{2}}^{-\nu}\left\{(2 x)^{\nu}\left(x^{2}-t^{2}\right)^{\lambda / 2} \mathrm{P}_{\nu}^{-\lambda}\left(\frac{t}{x}\right) H(x-t)\right\} & \\
& =\frac{H(x-t)}{\Gamma(\lambda-\nu+1)}(x-t)^{\lambda-\nu} .
\end{aligned}
$$

It follows from (3.10) with $\nu=0$ and (3.5) that (3.11) holds if either $\alpha \geqq 0$ and $\beta>0$ or if $\alpha \leqq 0$ and $\alpha+\beta>0$; and that (3.12) holds if either $\nu \leqq 0$ and $\lambda>-1$ or $\lambda>\nu-1 \geqq-1$. With $\alpha=-n, \beta=n+$ $\lambda+1, n=0,1,2, \cdots,(3.11)$ leads to the formula

$$
\frac{1}{\Gamma(\lambda+n+1)}\left(\frac{d}{d x^{2}}\right)^{n}(x-t)^{\lambda+n}=(2 x)^{-n}\left(x^{2}-t^{2}\right)^{\lambda / 2} \mathrm{P}_{n-1}^{-\lambda}\left(\frac{t}{x}\right)
$$

since $P_{-n}^{-\lambda}=P_{n-1}^{-\lambda}$. This is a counterpart to Rodrigues' formula.

4. The integral equation. We are now ready for the discussion of the integral equation

$$
\int_{a}^{x}\left(x^{2}-t^{2}\right)^{\lambda / 2} P_{\nu}^{-\lambda}\left(\frac{t}{x}\right) g(t) d t=f(x), \quad 0<a<x \leqq b,
$$

in which $g$ is the unknown function and $f$ is a given function. We shall seek solutions $g$ in $A_{0}$ and for the present do not say much about the nature of $f$ beyond stipulating that it shall possess a representation of the form (4.1). Later we give sufficient conditions under which this is the case.

In view of (3.3) we may assume $\nu \geqq-\frac{1}{2}$ in (4.1); indeed $-\nu-1>-\frac{1}{2}$ if $\nu<-\frac{1}{2}$. It is seen from (3.1) that

$$
\left(x^{2}-t^{2}\right)^{\lambda / 2} P_{\nu}^{-\lambda}\left(\frac{x}{t}\right)=(x-t)^{\lambda} F(x, t),
$$

where $F$ is a bounded continuous function of $x$ and $t$ for $a \leqq t \leqq x \leqq b$, and it follows that $\lambda>-1$ is a necessary and sufficient condition for the existence of the integral in (4.1) for each $g \in A_{0}$ and a.e. $x$. Accordingly, we assume

$$
\lambda>-1, \quad \nu \geqq-\frac{1}{2} .
$$

With these assumptions it can be proved by Fubini's theorem that $f \in A_{0}$ : later we shall restrict $f$ to a smaller class.

Under assumptions (4.2) the conditions for the application of (3.8) are satisfied, and we may rewrite the integral equation as

$$
f(x)=\int_{a}^{b} I_{x}^{\lambda-\nu}\left\{\frac{H(x-t)}{\Gamma(\nu+1)}(2 t)^{-\nu}\left(x^{2}-t^{2}\right)^{\nu}\right\} g(t) d t .
$$


If it is permissible to interchange the operation $I_{x}^{\lambda-\nu}$ with integration with respect to $t$, we may use (2.12) and obtain

$$
f(x)=I_{x}^{\lambda-\nu} I_{x^{2}}^{\nu+1}\left\{(2 x)^{-\nu-1} g(x)\right\} .
$$

We shall now show that this relation is indeed equivalent to (4.1).

If $\lambda=\nu,(2.4)$ and (3.4) show that (4.1) and (4.4) are identical. If $\lambda>\nu \geqq-\frac{1}{2}$, the operator $I_{x}^{\lambda-\nu}$ is an integral operator of the form (2.7). In this case the right hand sides of (4.3) and (4.4) are repeated integrals; and these repeated integrals are equal by Fubini's theorem. There remains the case $\nu>\lambda>-1$, when $I_{x}{ }^{\nu-\lambda}$ is an integral operator. In this case we form

$$
I_{x}{ }^{\nu-\lambda} f(x)=I_{x}{ }^{\nu-\lambda} \int_{a}^{b} H(x-t)\left(x^{2}-t^{2}\right)^{\lambda / 2} P_{\nu}{ }^{-\lambda}\left(\frac{x}{t}\right) g(t) d t .
$$

The right hand side is a repeated integral in which the order of integrations can be interchanged by Fubini's theorem. When this is done, one obtains by (3.9) and (2.4)

$$
I_{x}^{\nu-\lambda} f(x)=I_{x^{2}}^{\nu+1}\left\{(2 x)^{-\nu-1} g(x)\right\},
$$

a relation equivalent to (4.4).

The solution of (4.4), and hence of the integral equation (4.1), may now be written in the form

$$
g(x)=(2 x)^{\nu+1} I_{x^{2}}^{-\nu-1} I_{x}^{\nu-\lambda} f(x) .
$$

We shall now discuss the conditions that must be imposed on $f$ in order that (4.1) have a solution $g \in A_{0}$. We have already pointed out that $f \in A_{0}$ is a necessary condition. Let us set $h(x)=(2 x)^{-\nu-1} g(x)$. Since $g \in A_{0}$, also $h \in A_{0}$, and it follows from the properties of $I_{x^{n}}^{\alpha}$ that $I_{x^{2}}^{\nu+1} h(x) \in$ $A_{[\nu]+1}$. If, moreover $[\nu]+1+\lambda-\nu \geqq 0$, then $I_{x}^{\lambda-\nu} I_{x^{2}}^{\nu+1} h(x) \in A_{[\nu]+1+[\lambda-\nu]}$. We now see that a necessary condition for the existence of a solution $g \in A_{0}$ of (4.1) is that $f \in A_{j}$, where

$$
j=\max \{0,1+[\nu]+[\lambda-\nu]\} .
$$

On the other hand, we will show that a sufficient condition for the existence of a solution $g \in A_{0}$ of (4.1) is that $f \in A_{k}$, where

$$
k=1-[-\nu]-[\nu-\lambda] .
$$

Indeed, $k+\nu-\lambda \geqq 0$ since $-\nu \leqq \frac{1}{2}$ and $[-\nu] \leqq 0$. Consequently, $I_{x}{ }^{\nu-\lambda} f$ exists and belongs to $A_{k+[\nu-\lambda]}=A_{1-[-\nu]}$ if $f \in A_{k}$. Moreover, $1-[-\nu]$ $+(-\nu-1) \geqq 0$ and hence $g$, defined by (4.5), exists and belongs to $A_{0}$. That $g$ satisfies (4.1) follows by retracing the steps that lead from (4.1) to $(4.4)$. 
For any real number $\rho,[\rho]+[-\rho]=0$ if $\rho$ is an integer, and $[\rho]+[-\rho]$ $=-1$ if $\rho$ is not an integer. It follows that $j=k=1+\lambda$ if both $\nu$ and $\lambda$ are integers, i.e., the integral equation involves a derivative (of integral order) of Legendre's polynomial. In this case the condition $f \in A_{k}$ is both necessary and sufficient for the existence of an integrable solution of (4.1).

In all other cases $k-j=1$ or 2 , and our necessary and sufficient conditions differ. Although the gap between them could be narrowed somewhat by a more careful analysis of fractional integrals, the formulation of necessary and sufficient conditions seems to require a somewhat different approach and will be postponed to $\S 6$.

5. Explicit solutions. The explicit and analytic form of the solution (4.5) is different according as $\nu$ or $\lambda-\nu$ or both of these numbers are or are not nonnegative integers. In what follows, $m$ and $n$ will stand for nonnegative integers, and $k$ is defined by (4.7).

First assume $\lambda-\nu=m$. Since $\lambda-\nu<k$, we have $I_{x}{ }^{\nu-\lambda} f(x)=f^{(m)}(x)$ in this case by (2.4). If, in addition, also $\nu=n$, then (4.5) may be transcribed as

$$
g(x)=(2 x)^{n+1}\left(\frac{d}{d x^{2}}\right)^{n+1} f^{(m)}(x), \quad \lambda=m+n, \quad \nu=n .
$$

If $\nu$ is not an integer and $\nu<n$, where $n$ is an integer, then we may write $I_{x^{2}}^{-\nu-1}=I_{x^{2}}^{-n-1} I_{x^{2}}^{n-\nu}$ and have

$$
\begin{aligned}
& g(x)=\frac{(2 x)^{\nu+1}}{\Gamma(n-\nu)}\left(\frac{d}{d x^{2}}\right)^{n+1} \int_{a}^{x}\left(x^{2}-t^{2}\right)^{n-\nu-1} f^{(m)}(t) 2 t d t, \\
& \lambda=\nu+m, \quad \nu<n .
\end{aligned}
$$

Next we turn to nonintegral values of $\lambda-\nu$. Since $\lambda-\nu<k$, there will be a nonnegative integer $m$ so that $\lambda-\nu<m \leqq k$; indeed we may take $m=0$ if $\lambda \leqq \nu$, and $m=-[\nu-\lambda]$ if $\lambda>\nu$. We write $I_{x}^{\nu-\lambda}=I_{x}^{\nu-\lambda+m} I_{x}{ }^{-m}$ and have accordingly,

$$
I_{x}^{\nu-\lambda} f(x)=\frac{1}{\Gamma(\nu-\lambda+m)} \int_{a}^{x}(x-t)^{\nu-\lambda+m-1} f^{(m)}(t) d t .
$$

If, in this case, $\nu=n$ is an integer, we have from (4.5),

$$
\begin{aligned}
& g(x)=\frac{(2 x)^{n+1}}{\Gamma(m+n-\lambda)}\left(\frac{d}{d x^{2}}\right)^{n+1} \int_{a}^{x}(x-t)^{m+n-\lambda-1} f^{(m)}(t) d t, \\
& \nu=n, \quad m=0 \quad \text { if } \lambda<n, \quad m=-n-[-\lambda] \text { if } \lambda>n .
\end{aligned}
$$

Lastly, if $\nu$ is not an integer, choose $n$ so that $\nu<n$ and write 


$$
\begin{aligned}
& I_{x^{2}}^{-\nu-1}=I_{x^{2}}^{-n-1} I_{x^{2}}^{n-\nu} . \text { We have } \\
& I_{x^{2}}^{n-\nu}\left\{\frac{H(x-t)}{\Gamma(m+\nu-\lambda)}(x-t)^{m+\nu-\lambda}\right\} \\
& \quad=(2 x)^{n-\nu}\left(x^{2}-t^{2}\right)^{(m+n-\lambda-1) / 2} \mathrm{P}_{n-\nu}^{1-m-n+\lambda}\left(\frac{t}{x}\right) H(x-t)
\end{aligned}
$$

by (3.11), and this enables us to evaluate

$$
I_{x^{2}}^{n-\nu} I_{x}{ }^{\nu-\lambda} f(x)
$$

by interchanging the orders of integration. Using the result in (4.5), we finally obtain

$$
\begin{aligned}
& g(x)=(2 x)^{\nu+1}\left(\frac{d}{d x^{2}}\right)^{n+1} \\
& \cdot\left\{(2 x)^{n-\nu} \int_{a}^{x}\left(x^{2}-t^{2}\right)^{(m+n-\lambda-1) / 2} P_{n-\nu}^{1-m-n+\lambda}\left(\frac{t}{x}\right) f^{(m)}(t) d t\right\}, \\
& \quad \nu<n, \quad m=0 \text { if } \lambda \leqq \nu, \quad m=-[\nu-\lambda] \quad \text { if } \quad \lambda>\nu .
\end{aligned}
$$

It may be verified by means of (3.4) and (3.5) that (5.1), (5.2) and (5.3) are particular cases of (5.4); but on account of their simplicity it seems appropriate to list the former relations separately. It is noteworthy that a comparatively elementary solution is obtained whenever at least one of the numbers $\nu$ and $\lambda-\nu$ is a nonnegative integer. This is probably explained by the circumstance that these are precisely the cases when $P_{\nu}^{-\lambda}(z)$ is the product of an elementary function and a polynomial. For $\nu=n$ this can be seen from (3.1) and for $\lambda-\nu=m$, either from a familiar expansion $[8, \S 3.2(16)]$ or else from the formula connecting Legendre functions and ultraspherical polynomials [8, §3.15(4)].

6. Necessary and sufficient conditions. Necessary and sufficient conditions for the existence of an integrable solution of (4.1) can be based on the observation that the class of functions representable in the form $I_{x^{n}}^{\alpha} f(x)$, with a fixed $\alpha>0$ and some integrable $f$, is independent of $n$. We shall first establish this fact.

Let $\phi$ be an infinitely differentiable function, and assume that $\phi^{\prime}(x)>0$, on $[a, b]$. Repeated integrals with respect to $\phi$ may be defined by the formula

$$
I_{\phi}^{\alpha} f(x)=\frac{1}{\Gamma(\alpha)} \int_{a}^{x}[\phi(x)-\phi(t)]^{\alpha-1} f(t) \phi^{\prime}(t) d t, \quad \alpha>0,
$$

with the usual extension to $\alpha \leqq 0$.

Let us fix $\alpha>0$, and let us denote by $B_{\alpha}$ the class of functions representable in the form $I_{x}{ }^{\alpha} f$ with an integrable $f$. We shall prove that the class 
of functions representable in the form ${I_{\phi}}^{\alpha} f$ with an integrable $f$ is independent of $\phi$ and hence is $B_{\alpha}$.

Since the inverse, $\phi^{-1}$, of $\phi$ is again an infinitely differentiable function with a positive derivative, and therefore the relationship between the classes of functions representable in the forms $I_{\phi}{ }^{\alpha} f$ and $I_{x}{ }^{\alpha} f$ respectively is mutual, it will be sufficient to show that for integrable $f$,

$$
I_{\phi}^{\alpha} f=I_{x}^{\alpha} g,
$$

where $g$ is also integrable.

We shall first prove (6.2) for $0<\alpha<1$ by showing that

$$
I_{x}^{1-\alpha} I_{\phi}^{\alpha} f(x)=I_{x}^{1} g(x)=\int_{a}^{x} g(t) d t .
$$

Justifying the change in the order of integrations by Fubini's theorem, we have

$$
\begin{aligned}
I_{x}^{1-\alpha} I_{\phi}^{\alpha} f(x)=\frac{1}{\Gamma(\alpha) \Gamma(1-\alpha)} \int_{a}^{x}(x-u)^{-\alpha} \\
\cdot\left\{\int_{a}^{u}[\phi(u)-\phi(t)]^{\alpha-1} \phi^{\prime}(t) f(t) d t\right\} d u \\
=\int_{a}^{x} f(t)\left\{\frac{\phi^{\prime}(t)}{\Gamma(\alpha) \Gamma(1-\alpha)} \int_{t}^{x}(x-u)^{-\alpha}[\phi(u)-\phi(t)]^{\alpha-1} d u\right\} d t
\end{aligned}
$$

or

$$
I_{x}^{1-\alpha} I_{\phi}^{\alpha} f(x)=\int_{a}^{x} f(t) \Phi(t, x) d t
$$

where

$$
\Phi(t, x)=\frac{\phi^{\prime}(t)}{\Gamma(\alpha) \Gamma(1-\alpha)} \int_{t}^{x}(x-u)^{-\alpha}[\phi(u)-\phi(t)]^{\alpha-1} d u .
$$

Now,

$$
\phi(u)-\phi(t)=\int_{t}^{u} \phi^{\prime}(w) d w=(u-t) \int_{0}^{1} \phi^{\prime}(t+(u-t) v) d v
$$

and

$$
[\phi(u)-\phi(t)]^{\alpha-1}=(u-t)^{\alpha-1} \psi(t, u),
$$

where

$$
\psi(t, u)=\left[\int_{0}^{1} \phi^{\prime}(t+(u-t) v) d v\right]^{\alpha-1}
$$


is infinitely differentiable for $a \leqq t \leqq u \leqq b$. Hence

$$
\begin{aligned}
\Phi(t, x) & =\frac{\phi^{\prime}(t)}{\Gamma(\alpha) \Gamma(1-\alpha)} \int_{t}^{x}(x-u)^{-\alpha}(u-t)^{\alpha-1} \psi(t, u) d u \\
& =\frac{\phi^{\prime}(t)}{\Gamma(\alpha) \Gamma(1-\alpha)} \int_{0}^{1} v^{\alpha-1}(1-v)^{-\alpha} \psi(t, t+(x-t) v) d v
\end{aligned}
$$

is an infinitely differentiable function for $a \leqq t \leqq x \leqq b$, and

$$
\Phi(t, t)=\phi^{\prime}(t) \psi(t, t)=\left[\phi^{\prime}(t)\right]^{\alpha} .
$$

We then have

$$
\Phi(t, x)=\left[\phi^{\prime}(t)\right]^{\alpha}+\int_{t}^{x} \frac{\partial \Phi(t, u)}{\partial u} d u .
$$

Substituting this in (6.4), we once more interchange the orders of integration in the repeated integral to obtain

$$
\begin{aligned}
I_{x}^{1-\alpha} I_{\phi}{ }^{\alpha} f(x) & =\int_{a}^{x} f(t)\left[\phi^{\prime}(t)\right]^{\alpha} d t+\int_{a}^{x} f(t)\left\{\int_{t}^{x} \frac{\partial \Phi(t, u)}{\partial u} d u\right\} d t \\
& =\int_{a}^{x}\left\{f(t)\left[\phi^{\prime}(t)\right]^{\alpha}+\int_{a}^{t} f(u) \frac{\partial \Phi(u, t)}{\partial t} d u\right\} d t .
\end{aligned}
$$

This is of the form (6.3), with

$$
g(x)=f(x)\left[\phi^{\prime}(x)\right]^{\alpha}+\int_{a}^{x} f(u) \frac{\partial \Phi(u, x)}{\partial x} d u .
$$

Clearly, $g$ is integrable if $f$ is integrable; and if $f \in A_{n}$ for some $n$, then also $g \in A_{n}$. We have thus proved (6.2) for $0<\alpha<1$ and have shown that in the event that one of the two functions $f$ and $g$ is in $A_{n}$, the other function is also in $A_{n}$.

Next we note that (6.2) clearly holds when $\alpha=n$ is a positive integer, since in this case $B_{n}=A_{n}$ of $\S 2$. Lastly, let $\alpha=n+\rho, 0<\rho<1$. Then

$$
I_{\phi}{ }^{\alpha} f=I_{\phi}{ }^{\rho}\left(I_{\phi}{ }^{n} f\right)=I_{x}{ }^{\rho} h
$$

by (6.2). Also, $I_{\phi}{ }^{n} f \in A_{n}$, and hence $h \in A_{n}$ so that $h=I_{x}{ }^{n} h^{(n)}$, and

$$
I_{\phi}{ }^{\alpha} f=I_{x}{ }^{\rho}\left(I_{x}{ }^{n} h^{(n)}\right)=I_{x}{ }^{\alpha} h^{(n)},
$$

so that (6.2) holds with $g=h^{(n)}$. Moreover if $f \in A_{j}, j=0,1,2, \cdots$, then also $g \in A_{j}$ and vice versa.

We can now show that under the assumptions (4.2) a necessary and sufficient condition for (4.1) to possess an integrable solution is that $f \in B_{\lambda+1}$. 
Indeed if $g(x)$, and therefore also $(2 x)^{-\nu-1} g(x)$, is integrable, then

$$
I_{x^{2}}^{\nu+1}\left\{(2 x)^{-\nu-1} g(x)\right\}=I_{x}^{\nu+1} h(x),
$$

and by (4.4)

$$
f(x)=I_{x}^{\lambda-\nu} I_{x}^{\nu+1} h(x)=I_{x}^{\lambda+1} h(x),
$$

so that the condition is necessary. On the other hand, if $f(x)=I_{x}^{\lambda+1} h(x)$, then

$$
I_{x}^{\nu-\lambda} f(x)=I_{x}^{\nu+1} h(x)=I_{x}^{\nu+1} k(x),
$$

and by (4.5)

$$
g(x)=(2 x)^{\nu+1} I_{x^{2}}^{-\nu-1} I_{\nu}{ }^{\nu-\lambda} f(x)=(2 x)^{\nu+1} k(x) .
$$

This function is integrable and satisfies (4.1) so that the condition is also sufficient.

A simple characterization of $B_{\lambda+1}$ does not appear to be available except when $\lambda+1$ is an integer, and $B_{\lambda+1}=A_{\lambda+1}$. If $n$ is an integer, and $n \leqq \lambda$ $+1<n+1$, it is known that $f \in B_{\lambda+1}$ if and only if either $I_{x}{ }^{n-\lambda} f(x) \in A_{n+1}$ or else $f \in A_{n}$ and $I_{x}{ }^{n-\lambda} f^{(n)}(x) \in A_{1} \quad[6$, Theorem 4].

The work of this section leads to alternative explicit forms of the solution of (4.1). Since $f \in B_{\lambda+1}$, we have $f=I_{x}{ }^{\lambda+1} h$ for some integrable $h$, and the solution of the integral equation (4.1) can be expressed in terms of $h$ as follows. From (4.5),

$$
\begin{aligned}
g(x) & =(2 x)^{\nu+1} I_{x^{2}}^{-\nu-1} I_{x}{ }^{\nu+1} h(x) \\
& =(2 x)^{\nu+1} \frac{d}{d\left(x^{2}\right)} I_{x^{2}}^{-\nu} I_{x}{ }^{\nu+1} h(x) .
\end{aligned}
$$

Computations similar to those preceding (5.4) enable us to evaluate $I_{x^{2}}^{-\nu} I_{x}^{\nu+1} f(x)$ and lead to

$$
g(x)=(2 x)^{\nu} \frac{d}{d x}\left\{(2 x)^{-\nu} \int_{a}^{x} P_{-\nu}\left(\frac{t}{x}\right) h(t) d t\right\} .
$$

There is an obvious connection between this explicit solution and (5.4); indeed one may be converted into the other by fractional integration by parts. If $\lambda=m-1$ is an integer, then (5.4) with $n=0$ becomes (6.6).

Note added September, 1963. Since this paper was written, some additional references have come to my notice. R. G. Buschman [10] solves the same integral equation as Higgins [3]. His method differs from those employed by Higgins and the present paper. K. N. Srivastava [11] solves certain integral equations which can be reduced to an equation that is a counterpart 
of (1.4), viz., to the integral equation

$$
\begin{aligned}
\int_{x}^{\infty}\left(t^{2}-x^{2}\right)^{\lambda / 2} \mathrm{P}_{\nu}^{-\lambda}\left(\begin{array}{l}
x \\
t
\end{array}\right) g(t) d t & =f(x), \\
\lambda & >-1, \quad \nu \geqq-\frac{1}{2}, \quad 0<a \leqq x .
\end{aligned}
$$

Srivastava's solution (which is based upon an application of Fourier and Hankel transforms) appears to be in error, but the integral equation can be solved by the methods of the present paper in the form

$$
g(x)=(2 x)^{\nu+1} K_{x^{2}}^{-\nu-1} K_{x}^{\nu-\lambda} f(x),
$$

where $K^{\alpha}$ is the operator of fractional integration defined, for $\alpha>0$ and suitable $f$, by

$$
K_{x^{n}}^{\alpha} f(x)=\frac{1}{\Gamma(\alpha)} \int_{x}^{\infty}\left(t^{n}-x^{n}\right)^{\alpha-1} f(t) n t^{n-1} d t .
$$

This definition is then extended, analogously to the work of $\$ 2$, to other values of $\alpha$.

\section{REFERENCES}

[1] TA LI, A new class of integral transforms, Proc. Amer. Math. Soc., 11 (1960), pp. 290-298.

[2] R. G. Buschman, An inversion integral for a Legendre transformation, Amer. Math. Monthly, 69 (1962), pp. 288-289.

[3] T. P. Hrgarss, An inversion integral for a Gegenbauer transformation, this Journal, 11 (1963), pp. 886-893.

[4] A. ERd Elly , An integral equation involving Legendre's polynomial, Amer. Math. Monthly, 70 (1963), pp. 651-652.

[5] H. KoBer, On a theorem of Schur and on fractional integrals of purely imaginary order, Trans. Amer. Math. Soc., 50 (1941), pp. 160-174.

[6] J. D. Tamarkin, On integrable solutions of Abel's integral equation, Ann. of Math., 31 (1930), pp. 219-229.

[7] G. H. HaRdy and J. E. LitTlewood, Some properties of fractional integrals. I., Math. Zeitschr., 27 (1927-28), pp. 565-606.

[8] A. ERdélyi et al., Higher Transcendental Functions, 3 volumes, McGraw-Hill, New York, 1953-1955.

[9] A. Erdélyi et aL., Tables of Integral Transforms, 2 volumes, McGraw-Hill, New York, 1954.

[10] R. G. Buschman, An inversion integral, Proc. Amer. Math. Soc., 13 (1962), pp. 675-677.

[11] K. N. SRivastava, On some integral transforms, Math. Japon., 6 (1961-62), pp. 65-72. 